This item was submitted to Loughborough's Research Repository by the author.

Items in Figshare are protected by copyright, with all rights reserved, unless otherwise indicated.

\title{
A 3D-printed polymer micro-gripper with self-defined electrical tracks and thermal actuator
}

PLEASE CITE THE PUBLISHED VERSION

https://doi.org/10.1088/1361-6439/aa631e

\section{PUBLISHER}

(C) IOP Publishing

\section{VERSION}

VoR (Version of Record)

\section{PUBLISHER STATEMENT}

This work is made available according to the conditions of the Creative Commons Attribution 3.0 Unported (CC BY 3.0) licence. Full details of this licence are available at: http://creativecommons.org/licenses/by/3.0/

\section{LICENCE}

CC BY 3.0

\section{REPOSITORY RECORD}

Alblalaihid, Khalid, J.K. Overton, Simon Lawes, and Peter Kinnell. 2019. "A 3d-printed Polymer Micro-gripper with Self-defined Electrical Tracks and Thermal Actuator”. figshare. https://hdl.handle.net/2134/24999. 
A 3D-printed polymer micro-gripper with self-defined electrical tracks and thermal actuator

This content has been downloaded from IOPscience. Please scroll down to see the full text.

2017 J. Micromech. Microeng. 27045019

(http://iopscience.iop.org/0960-1317/27/4/045019)

View the table of contents for this issue, or go to the journal homepage for more

Download details:

IP Address: 158.125.80.91

This content was downloaded on 08/06/2017 at 14:49

Please note that terms and conditions apply.

You may also be interested in:

Polymer micro-grippers with an integrated force sensor for biological manipulation

R E Mackay, H R Le, S Clark et al.

FEM and experimental characterization of silicon-polymer micro gripper

F Krecinic, T Chu Duc, G K Lau et al.

Comparison of microtweezers based on three lateral thermal actuator configurations

J K Luo, A J Flewitt, S M Spearing et al.

An experimentally verified model for thermal microactuators including nonlinear material properties, vacuum, and intra-device heat conduction

Keegan Colbert, Mohammad Naraghi and James G Boyd

On heat transfer at microscale with implications for microactuator design

Ozgur Ozsun, B Erdem Alaca, Arda D Yalcinkaya et al.

An optimal design of thermal-actuated and piezoresistive-sensed CMOS-MEMS resonant sensor

Chun-Chieh Wang, Chun-Yin Tsai, Tsung-Lin Chen et al.

An in-plane thermal unimorph using confined polymers

G K Lau, T Chu Duc, J F L Goosen et al.

Distributed and lumped element models for a bimorph-actuated micromirror

Sagnik Pal and Huikai Xie 


\title{
A 3D-printed polymer micro-gripper with self-defined electrical tracks and thermal actuator
}

\author{
Khalid Alblalaihid ${ }^{1}$, James Overton ${ }^{2}$, Simon Lawes ${ }^{2}$ and Peter Kinnell ${ }^{3}$ \\ ${ }^{1}$ National Center for Aeronautical Technology, King Abdulaziz City for Science and Technology \\ (KACST), Riyadh 11442, Saudi Arabia \\ ${ }^{2}$ Manufacturing Metrology Team, Advanced Manufacturing Metrology Research Group, Faculty \\ of Engineering, The University of Nottingham, Nottingham NG7 2RD, United Kingdom \\ ${ }^{3}$ EPSRC Centre for Innovative Manufacturing in Intelligent Automation, Wolfson School of Mechanical \\ and Manufacturing Engineering, Loughborough University, Loughborough LE11 3UZ, United Kingdom
}

E-mail: P.kinnell@lboro.ac.uk

Received 26 December 2016, revised 20 February 2017

Accepted for publication 27 February 2017

Published 17 March 2017

\begin{abstract}
This paper presents a simple fabrication process that allows for isolated metal tracks to be easily defined on the surface of 3D printed micro-scale polymer components. The process makes use of a standard low cost conformal sputter coating system to quickly deposit thin film metal layers on to the surface of 3D printed polymer micro parts. The key novelty lies in the inclusion of inbuilt masking features, on the surface of the polymer parts, to ensure that the conformal metal layer can be effectively broken to create electrically isolated metal features. The presented process is extremely flexible, and it is envisaged that it may be applied to a wide range of sensor and actuator applications. To demonstrate the process a polymer micro-scale gripper with an inbuilt thermal actuator is designed and fabricated. In this work the design methodology for creating the micro-gripper is presented, illustrating how the rapid and flexible manufacturing process allows for fast cycle time design iterations to be performed. In addition the compatibility of this approach with traditional design and analysis techniques such as basic finite element simulation is also demonstrated with simulation results in reasonable agreement with experimental performance data for the micro-gripper.
\end{abstract}

Keywords: 3D-printed, micro-gripper, thermal actuator

(Some figures may appear in colour only in the online journal)

\section{Introduction}

Over the last decade there have been significant advances in the field of additive manufacturing. Technology that was initially referred to as a rapid prototyping method is increasingly finding possible applications in mainstream manufacturing. For example, additive manufacturing is being applied to the production of complex high performance components for aerospace applications, or bespoke patient specific medical devices $[1,2]$. The technology has also developed to allow the creation of miniature scale parts, with high-precision geometry that may be suitable for applications such as sensors or micro actuators. The available fabrication tools allow for micro scale devices to be fabricated with features ranging from tens of millimeters [3], down to less than $100 \mathrm{~nm}$ using the latest two-photon polymerization techniques [4-6]. In this paper the possibility to apply additive manufacturing technology on the micro-scale, for the production of complex micro-electro-mechanical devices, is presented. A commercially available 3D Projection-microstereolithography (PMSL) system manufactured by Envisiontec was used to demonstrate that it is possible to create devices with dimensions and complexity that approach that of traditional MEMS (micro-electro-mechanical system) structures. 
The PMSL process offers significant design freedom as compared to many MEMS processes. For example, it allows true $3 \mathrm{D}$ structures to be fabricated in a single step, instead of the typically 2.5D layer based structures that typify many MEMS devices. A further benefit is that the process does not require the time consuming fabrication of expensive photomasks, instead parts are created directly from a CAD definition. The PMSL process has previously been applied to the manufacture of a number of MEMS devices; for example, in previous work it has been shown that the PMSL process can be used to manufacture complex single material MEMs sensor packaging structures [7]. In another interesting example, a polymer micro-gripper based on a hydraulically actuated micro-bellows was created [8]. While the potential to create useful geometry is clear, there are two clear limitations as compared to traditional MEMS processing. First, devices are fabricated by serial processing or in low batch sizes of only a few units at a time. While this may prohibit the technology from supporting high volume production, the technology is well suited to the production of highly customised devices in a short timescale. Second, a significant limitation of the process is the inability to create multi-material devices; to address this, previous work has been done using modified polymers to enhance the polymer material properties, allowing the creation of miniature flow sensors [9]. There are also examples of the PMSL process being combined with a post-build metallization step, to produce a conformal metal layer over a polymer parts, making parts electrically conducting [10]. To create MEMS devices that incorporate metallic electrical tracks, a metal deposition step followed by an additional selective polishing process has also been presented [11]. The problem with this approach is that post processing requires complicated fixtures to hold the micro part, and high precision processing is required to selectively remove the metal layer without damaging the underlying polymer structure.

In this paper, the development of a simple process that allows complex isolated metal tracks to be created on polymer micro parts, without the need for post processing of deposited metal layers, is presented. The resulting process allows the rapid fabrication of micro devices with integral electrical tracks, which are created without the need of tooling or complex post metal deposition processing. The process and associated design guidelines are first presented in section 2 , then applied to demonstrate how a thermally actuated polymer micro-gripper can be created using a 3D printing approach. Process characterization techniques and design methodologies that exploit the ability of the PMSL process to create evaluation parts in a matter of hours are presented. In addition the ability to use finite element simulation to predict device performance is demonstrated and verified by comparison with experimental test data collected from a fabricated gripper.

\section{Fabrication process}

The basis for the work described in this paper, is the manufacture of polymer parts using the well-established PMSL process. The fabrication of isolated metal tracks, without
Step 1 PMSL process, each layer is $25 \mu \mathrm{m}$ thick

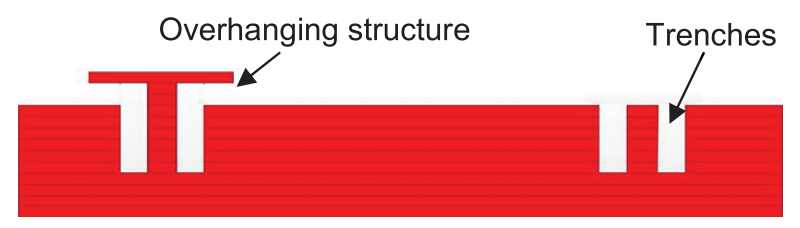

Step 2 Sputter coated metal layer deposition

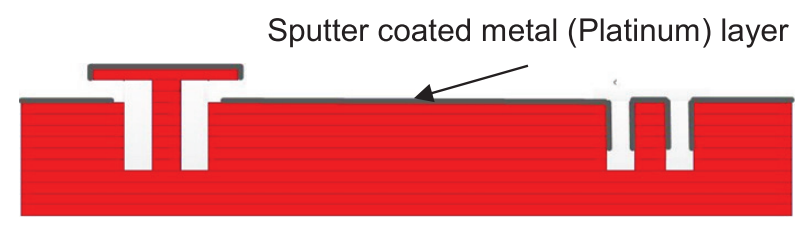

Figure 1. Illustration of the simple two-step process to create electrical path using overhanging structure or tranches. (a) The first step is to create the device using micro-stereolithography by sequentially building a stack of $25 \mu \mathrm{m}$ thick layers; (b) the second step is to metalize the device with a metal layer using sputter coating.

the need for any post metallization processing, is achieved by using a simple design feature to create inbuilt masking structures on the surface of the produced polymer parts. At the design stage these features are included on the polymer part in response to the desired configuration of metal track and features. The objective of the masking structures is to limit the effectiveness of the subsequent metal deposition process, and therefore prevent the full conformal coating of the surface with a metal layer. The process therefore makes use of two main steps as illustrated in figure 1; step one is the microstereo-lithography stage, for the production of the polymer component; step two is the conformal thin film metal deposition step. To illustrate the possibilities of the process a series of test geometries designed to be electrically isolated have been presented in previous work [12], these include micro electrical probe needles fabricated on isolated contact pads.

Step one is a solid-freeform fabrication technique, and is based on the spatially controlled solidification of a liquid and light-sensitive resin upon interaction with a light source (photopolymerisation). An LCD or a digital micromirror device (DMD) is used to layer profiles according to data from an STL (stereo lithography format) file. In this way $25 \mu \mathrm{m}$ layers of polymer are successively exposed and solidified to build up 3D geometries. All the polymer parts created in this work were built on an Envisiontec Perfactory Mini-Multi Lens projection-microstereolithography machine; using the R11 rapid-prototyping material. This resin was chosen due to its well established curing behavior in the build process and material properties that match the requirements in the envisaged MEMS applications (a thermally actuated microgripper); such as a relatively high heat resistance capability and a tensile strength of 54.0 MPa [13]. The brightness of the stereolithography projector unit was calibrated to a light intensity of $600 \mathrm{~mW} \mathrm{dm} \mathrm{m}^{-2}$ and the exposure time was set to $4.0 \mathrm{~s}$ per layer (standard range) at a layer height of $25 \mu \mathrm{m}$. The resolution $(X / Y)$ was set to $1050 \times 1400$ pixels $(\mathrm{SXGA}+)$, 
which corresponded to a pixel size of 10 micrometers. The parts were cleaned post build in an ultrasonic isopropanol bath for $3 \mathrm{~min}$, air-dried and then cut away from the support structure. All parts were visually inspected and measured on a Zeiss Stemi C-2000 optical microscope and a Hitachi E-2600N scanning-electron microscope in backscattered electron mode (SEM-BSE).

Step 2, the metal deposition, can be carried out using a wide range of deposition techniques. An obvious choice would be the use of an electron beam evaporation process, which due to the highly directional 'line-of-sight' nature of the deposition, would be easily hampered by the inbuilt masking structures [14]. A less ideal process, but more readily available, is sputter coating. This is typically used to prepare samples for imaging in a scanning electron microscope, and as such, it is able to provide conformal coatings over highly structured surfaces. Even though the metal layers produced by sputter coating are much more conformal than those produced by evaporation processes, if the correct masking structures are created, it is still possible to create isolated metal tracks and features. To demonstrate this, in this work metal was deposited using a standard low-cost Quorum sputter coating system. The design and optimization process followed to create the ideal masking structures for a given metal deposition process are described in section 3 .

The Quorum sputter coating system was used to deposit thin film Platinum layers with thickness ranging from 10 to $100 \mathrm{~nm}$, however, the system used in this work did not have a closed loop means to control film thickness. Therefore thickness was controlled by varying the duration of sputter coating, and estimated values of the thickness of deposited layers determined after the layers were deposited. This was done by measuring the layer thickness deposited on a small piece of polished Silicon wafer placed next to the polymer parts in the sputter coating machine, this process is described in more detail in section 3 . The process settings used for sputter coating were as follows: chamber pressure of 10-1 mbar, with a deposition current of between 15-20 mA.

\section{Test structures for mask structure optimisation}

The inbuilt masking features incorporate high aspect ratio trenches and overhanging structures that are designed to be difficult to cover with an unbroken metal layer. The effect of the high aspect ratio overhanging structures is to limit the transport of gas into the trenches and therefore limit the deposition of the metal in these areas. This is schematically demonstrated in figure 1, with an arrow indicating the trenches or overhangs that define effective metal isolation areas. To optimize the metal isolation features, a number of test structures were created. The objective of the test structures is to allow the metalisation process being used to be quickly characterised, leading to the formation of suitable design guidelines; these guidelines ensure that the conformal metal layer can be effectively defined as electrically isolated metal features. The test structures are simple metal pads that should be electrically isolated from the rest of the test substrate, and are designed

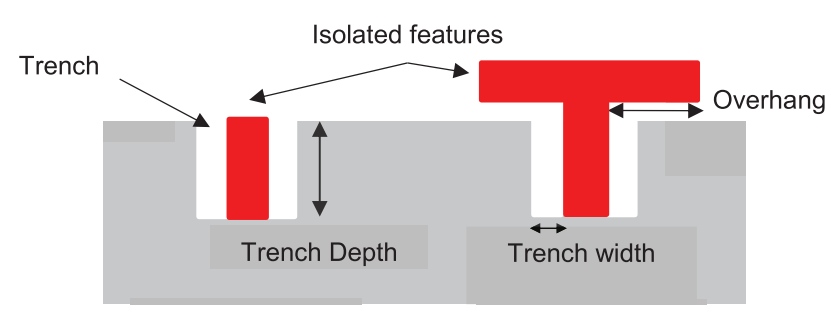

(a)

(b)

Figure 2. The cross-section of the test feature variants: (a) trenches structure and (b) overhanging structure.

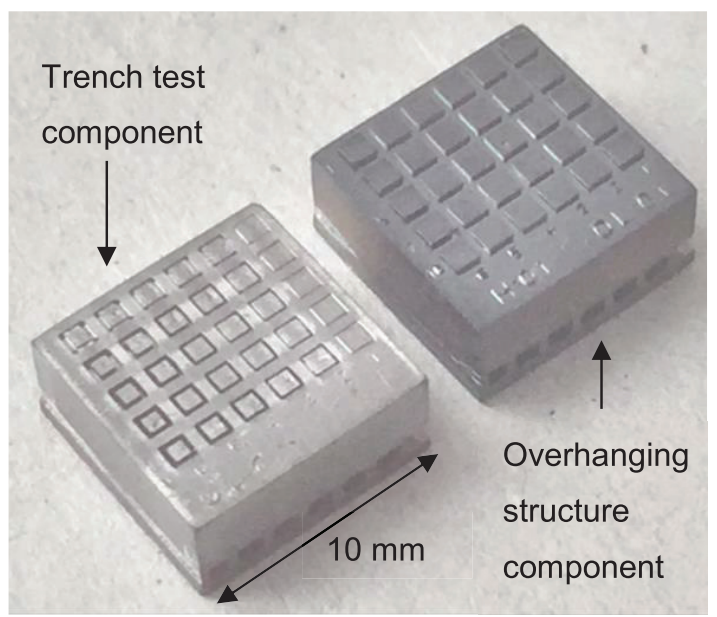

Figure 3. Metalized test components (tranches and overhanging structure component) shown next to a pen for scale.

to allow easy electrical probing. Figure 2 illustrates the two types of isolation feature that were considered; for each type an array of test structures was created with varying parameters to include a range of trench widths, depths and overhangs. Once metal is applied to the array of test features, each feature can then be probed to determine if it has remained electrically isolated.

A photograph of the two arrays of isolation structures with various configurations can be seen in figure 3. Test features on both test components had different dimensions, where trench width and depth were varied from $25 \mu \mathrm{m}$ to $150 \mu \mathrm{m}$ and $100 \mu \mathrm{m}$ to $1000 \mu \mathrm{m}$, respectively. As coating time is linked to metal layer thickness deposited, the layer thickness has been assessed by taking a measurement of the layer thickness every 90 s. To determine the layer thickness, a piece of polished silicon wafer was masked using PTFE tape and placed in the metal deposition system in close proximity to the test samples. The silicon test pieces were then removed, and the PTFE tape was also removed to reveal an uncoated area directly next to the deposited metal layer. The resulting step height was measured using a Bruker ContourGT-I measurement microscope. Layer thicknesses were measured to be $19 \mathrm{~nm}, 106 \mathrm{~nm}$ and $163 \mathrm{~nm}$ for coating times of $90 \mathrm{~s}, 360 \mathrm{~s}$ and $630 \mathrm{~s}$ respectively.

After depositing metal on both types of test structures, the pads on each were tested to check for electrical isolation. For both test components results are shown in tables 1-3, that were obtained for coating times of 90, 360 and 630 s respectively. The matrix represented in these three tables represents the 
Table 1. The electrical isolation results for (a) overhanging and (b) without overhanging structure at 90 s (coating time). ( $\checkmark$ ) is passed the isolation test and $(\mathbf{X})$ is failed the isolation test.

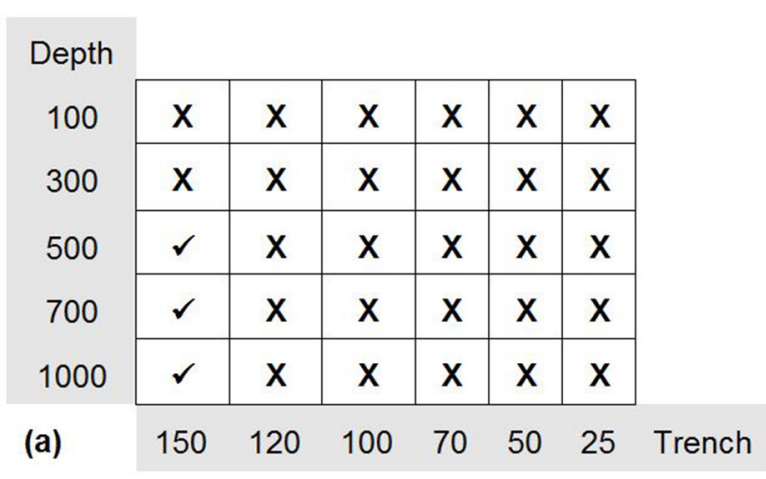

\begin{tabular}{|c|c|c|c|c|c|c|}
\hline \multirow{2}{*}{$\begin{array}{c}\text { Depth } \\
100\end{array}$} & \multirow[b]{2}{*}{$x$} & \multirow[b]{2}{*}{$x$} & \multirow[b]{2}{*}{$x$} & \multirow[b]{2}{*}{$x$} & \multirow[b]{2}{*}{$x$} & \multirow[b]{2}{*}{$x$} \\
\hline & & & & & & \\
\hline 300 & $x$ & $x$ & $x$ & $x$ & $x$ & $x$ \\
\hline 500 & $x$ & $x$ & $x$ & $x$ & $x$ & $x$ \\
\hline 700 & $x$ & $x$ & $x$ & $\checkmark$ & $x$ & $x$ \\
\hline 1000 & $x$ & $\checkmark$ & $x$ & $\checkmark$ & $x$ & $x$ \\
\hline (b) & 150 & 120 & 100 & 70 & 50 & 25 \\
\hline
\end{tabular}

Table 2. The electrical isolation results for (a) overhanging and (b) without overhanging structure at $360 \mathrm{~s}$ (coating time). ( $\checkmark$ ) is passed the isolation test and $(\mathbf{X})$ is failed the isolation test.

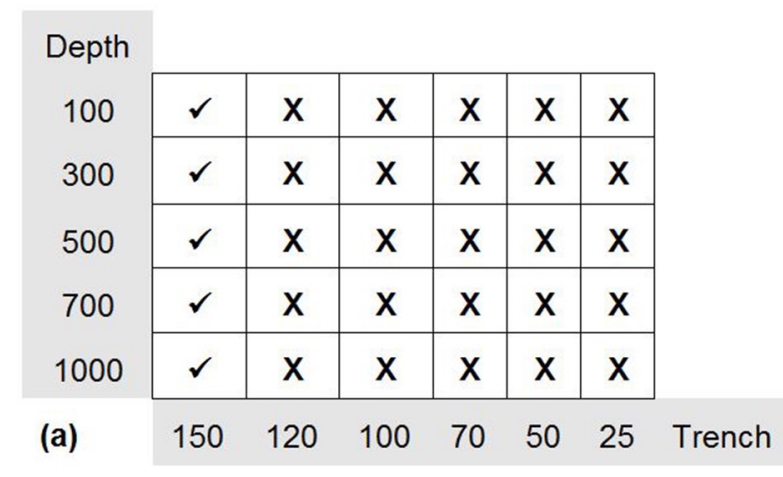

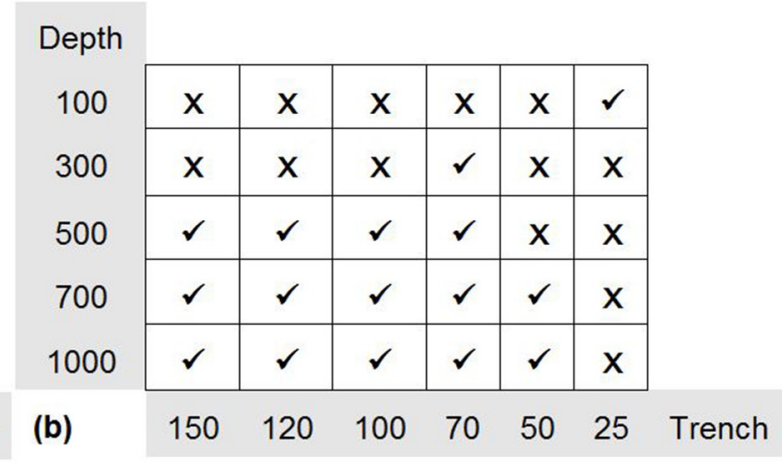

Table 3. The electrical isolation results for (a) overhanging and (b) without overhanging structure at 630s (coating time). ( $\checkmark$ ) is passed the isolation test and $(\mathbf{X})$ is failed the isolation test.

\begin{tabular}{|c|c|c|c|c|c|c|}
\hline \multirow{2}{*}{$\begin{array}{c}\text { Depth } \\
100\end{array}$} & & & & & & \\
\hline & $x$ & $x$ & $x$ & $x$ & $x$ & $x$ \\
\hline 300 & $x$ & $x$ & $x$ & 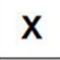 & $x$ & $x$ \\
\hline 500 & $\checkmark$ & $x$ & $x$ & $x$ & $x$ & $x$ \\
\hline 700 & $\checkmark$ & $x$ & $x$ & $x$ & $x$ & $x$ \\
\hline 1000 & $\checkmark$ & $x$ & $x$ & $x$ & $x$ & $x$ \\
\hline (a) & 150 & 120 & 100 & 70 & 50 & 25 \\
\hline
\end{tabular}

array of isolation structures present on the two test structures, where columns represent trench width, and rows represent trench depth. Electrical isolation tests were performed using a handheld multi-meter to check the connectivity between the feature and the pad base.

The first structure that was considered was the simple trench type without overhanging structures, as shown in figure 2(a). For these features it is clear that at $90 \mathrm{~s}$ coating time most pads were electrically isolated. However, the performance of the pads deteriorated significantly when coating time was increased to $360 \mathrm{~s}$, where generally the deeper the

\begin{tabular}{|c|c|c|c|c|c|c|}
\hline \multirow{2}{*}{$\begin{array}{c}\text { Depth } \\
100\end{array}$} & & & & & & \\
\hline & $x$ & $x$ & $x$ & $x$ & $x$ & $x$ \\
\hline 300 & $x$ & $x$ & $x$ & $x$ & $x$ & $x$ \\
\hline 500 & $x$ & $x$ & $x$ & $x$ & $x$ & $x$ \\
\hline 700 & $x$ & $x$ & $x$ & $\checkmark$ & $x$ & $x$ \\
\hline 1000 & $x$ & $\checkmark$ & $x$ & $\checkmark$ & $x$ & $x$ \\
\hline (b) & 150 & 120 & 100 & 70 & 50 & 25 \\
\hline
\end{tabular}

trench the more likely it is to provide isolation. In addition it can be noticed that the narrow trenches provide poor isolation which is thought to be a results of the increased likelihood of manufacturing faults causing bridging features to break the isolation. Nevertheless this simple to manufacture trench type isolation feature is suited for low thickness deposition of the metallic layers using the specified sputter coating system.

The second structure that was considered was the structure with the inbuilt overhang, as shown in figure 2(b). For the $90 \mathrm{~s}$ deposition time it can be seen that only the widest 


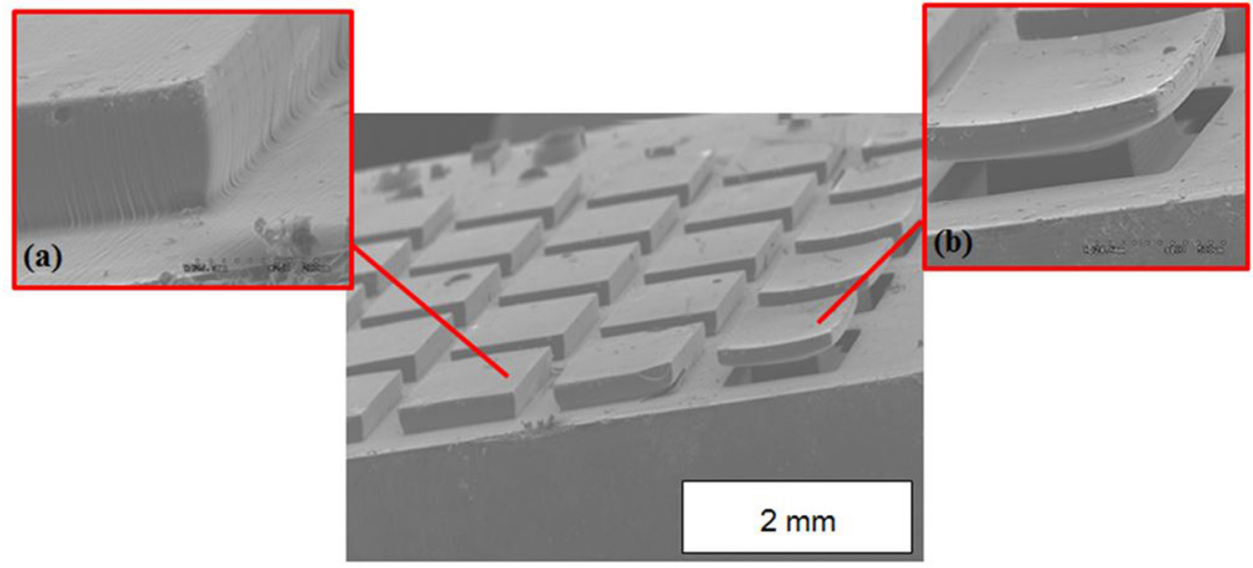

Figure 4. SEM image showing the overhanging structure: (a) the feature was not manufactured correctly as trenches were blocked with semi-cured resin, (b) the isolated feature bends due to gravity.

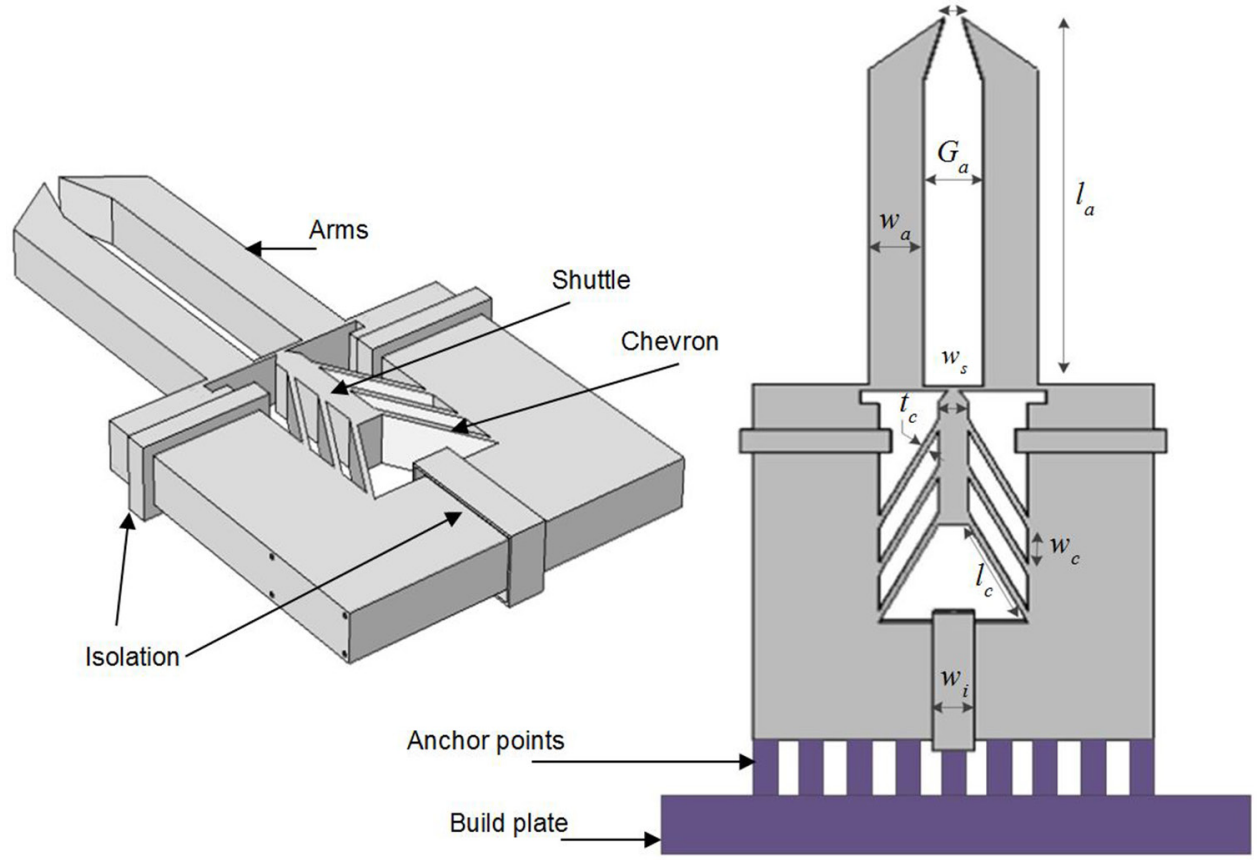

Figure 5. Thermally actuated micro-gripper, (a) with main structural elements labelled and (b) main geometric parameters labelled.

$(150 \mu \mathrm{m})$ trenches were isolated. As the intended design aim of the overhang was to improve isolation this result was unexpected. Further analysis of the manufactured test structure was conducted by inspection in a scanning electron microscope; this was able to determine that the cause of the poor isolation was due to a manufacturing limitation of the process that limits the ability to create large overhangs. It is believed that the liquid resin used was unable to drain from the structure during the build process, therefore blocking the trenches with semi-cured resin as can be seen in figure 4(a). In spite of this issue it was also observed that overhanging structures that were built above the trenches with a width of $150 \mu \mathrm{m}$ were manufactured with sufficient clearance to prevent metal shorting as can be seen in figure 4(b). Comparison of the $150 \mu \mathrm{m}$ trenches illustrates that the overhang feature is able to enhance isolation significantly.
Table 4. Geometric parameters of thermal micro-gripper.

\begin{tabular}{ll}
\hline Item & Specifications \\
\hline Arm & $l_{\mathrm{a}}=11 \mathrm{~mm}, g_{\mathrm{a}}=540 \mu \mathrm{m}, G_{\mathrm{a}}=1730 \mu \mathrm{m}, w_{\mathrm{a}}=1600 \mu \mathrm{m}$ \\
Shuttle & $w_{\mathrm{s}}=860 \mu \mathrm{m}$, \\
Chevron & $l_{\mathrm{c}}=3450 \mu \mathrm{m}, t_{\mathrm{c}}=180 \mu \mathrm{m}, w_{\mathrm{c}}=1080 \mu \mathrm{m}$ \\
Isolation & $w_{\mathrm{i}}=1200 \mu \mathrm{m}$, depth $=350 \mu \mathrm{m}$, trenches $=200 \mu \mathrm{m}$
\end{tabular}

\section{Design and simulation of a thermally actuated micro-gripper}

Micro grippers can be used for a wide range of manipulation and assembly tasks, and there are many examples of micro-grippers created using a wide range of manufacturing processes [15-17]; for more examples of micro gripper technology, a good review of micro grippers for robotic applications is given by [18]. The wide variety of gripper designs 


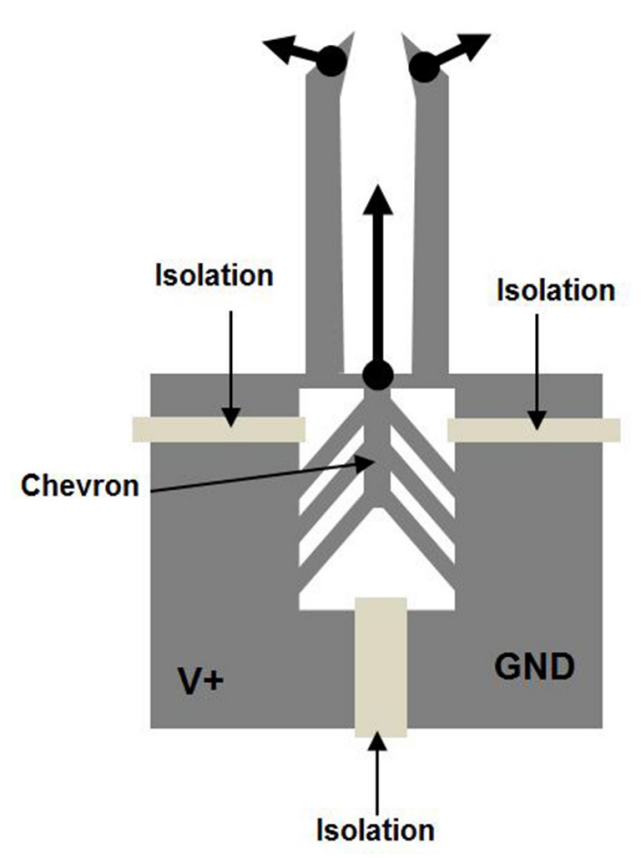

Figure 6. Micro actuator showing three isolation structures that create a current path through the chevron structure in the centre of the actuator, allowing supply voltage $V+$ to be applied to one side of the actuator while the other side is grounded.

presented in the literature illustrates the need for grippers to be fabricated with variable geometry to meet the varied needs of each application they are applied to. The flexibility offered by $3 \mathrm{D}$ printing technologies is therefore attractive from the perspective of providing the design freedom to create customized grippers with highly variable geometry.

The test structures presented in the previous section demonstrate the ability of the proposed inbuilt masking structures to create electrically isolated tracks, pads, and other metal features. Using the test structures it is also possible to define the design guidelines to allow the creation of suitable masking features. Based on the result from the test structures a thermally actuated 3D-printed polymer micro-gripper was designed. The design is similar in essences to many traditional MEMS micro grippers; the micro-grippers function is based on a combination of simple mechanical beam and flexure elements, and electrical heaters to generate local thermal expansion [16]. In MEMS devices electrostatic actuators based on comb-drives are also common [17]. It is envisaged that electrostatic actuators and transducers could be manufactured by the proposed process; however, this work focuses on the use of thermal actuation.

When designing traditional MEMS devices, often complicated multi-physics finite element simulations are favored. The large cost and lengthy fabrication times, associated with producing MEMS devices, make it is advantageous to focus efforts on detailed design simulations rather than fabricating large numbers of prototype batches. One of the main advantages offered by the additive manufacturing route is that prototype devices can be fabricated very quickly with no associated tooling costs; however, even though prototypes can be produced quickly the ability to evaluate possible device designs with a simple model will always be attractive. In this
Table 5. Material properties used in the simulation.

\begin{tabular}{ll}
\hline Material properties & Photopolymer \\
\hline Young's modulus $(E)$ & $2(\mathrm{GPa})[24]$ \\
Poisson's ratio $(v)$ & $0.3[24]$ \\
Thermal conductivity & $0.142 \mathrm{~W} \mathrm{~m}^{-1} \mathrm{~K}^{-1}[25]$ \\
Coefficient of thermal expansion & $120\left(\mu \mathrm{m} \mathrm{m}^{-1} \mathrm{~K}^{-1}\right)[25]$ \\
Density $(\rho)$ & $1219\left(\mathrm{Kg} \mathrm{m}^{-3}\right)[13]$ \\
\hline
\end{tabular}

work the use of simple finite element modelling was explored to provide a first approximation of device performance. The aim was to set up a basic finite element modelling capability using a limited set of simple elements. To model a thermal actuator the elements chosen would only have to include electrical and heat transfer capability, in addition to standard linear elastic material properties.

Based on the analysis of the test structures detailed in section 3, overhanging structures were used to create electrically isolated tracks running on the surface of a micro-scale gripper. The coating time was set to be less than $90 \mathrm{~s}$ and the width and depth, of the overhangs, were set to be $200 \mu \mathrm{m}$ and $350 \mu \mathrm{m}$ respectively to avoid poor isolation.

The key components of the designed micro-scale gripper and the geometric parameters that define the structures are shown in figure 5 , with the associated values given in table 4 . The isometric view of the gripper illustrates the actual structure that was built using the PMSL process, in the side view the gripper is shown located on a build plate. To attach the structure to the build plate, a set of thin anchor points were used; these can be easily cut to facilitate the safe removal of the gripper from the build plate.

The gripper is designed such that a current path is created from one side of the device to the other, running across the device via the chevron structure as shown in figure 6 . The relative geometry of the chevron as compared to the rest of the gripper ensures that the chevron represents the main resistance in the current path. As a potential difference is applied across the gripper, the resulting current flow causes resistive heating in the chevron. As the chevron heats it expands and a reaction force between the ends of the chevron and the main body of the gripper is created. The associated bending of the chevron beams forces the centre of the beams to be pushed up, indicated by the bold arrow in figure 6 . In turn, the bending created at the base of the gripper arms results in a rotation of each arm in opposite directions causing the arms to splay apart.

\subsection{Numerical simulation results}

The performance of the micro-gripper was modelled using ABAQUS finite element software. Material properties used in the model are detailed in table 5. Due to a lack of published data regarding the R11 polymer used in this work, material properties are based on values published for similar photosensitive resins.

The first step in the analysis was to model the temperature distribution derived from a coupled thermal-electric analysis. The element types that have been used in this analysis 


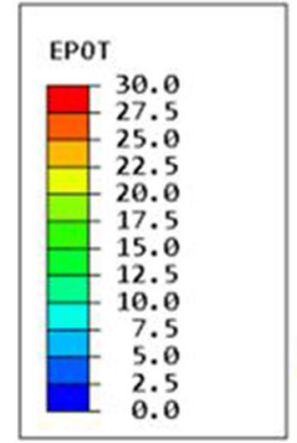

(a)

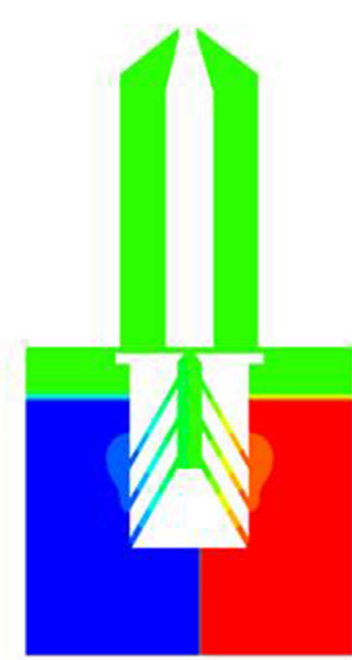

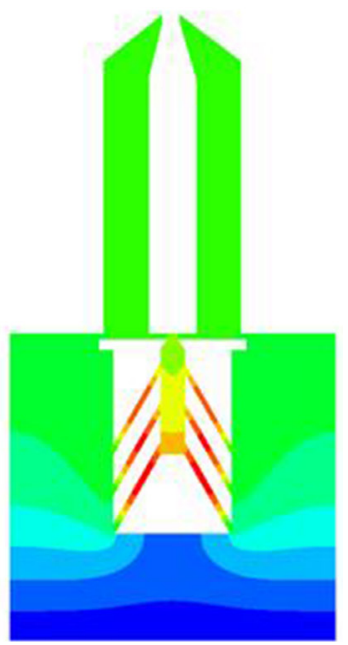

Figure 7. Result of thermal-electric coupled analysis subject to $30 \mathrm{~V}$ applied voltage and $22{ }^{\circ} \mathrm{C}$ sink temperature: (a) the distribution of electrical potential shown in volts, (b) the temperature profile shown in degrees Kelvin.

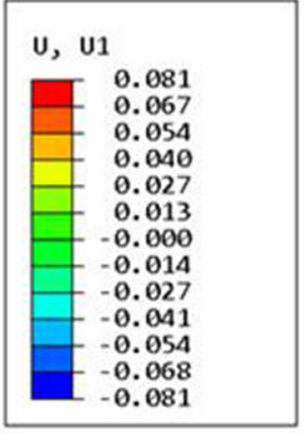

(a)

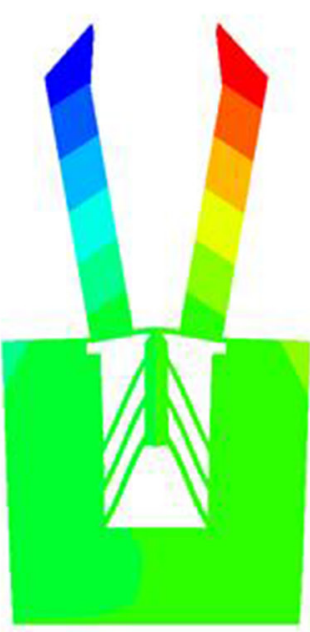

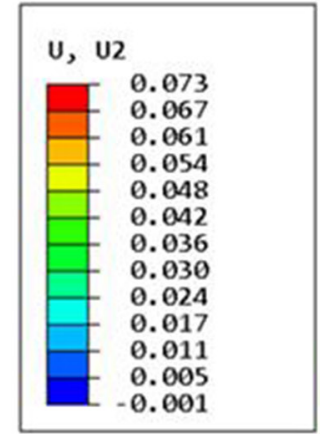

(b)

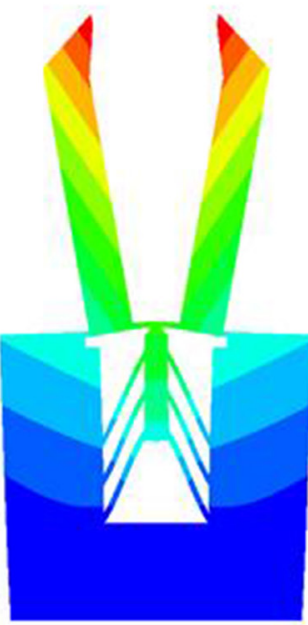

Figure 8. The in-plane displacement result of thermal structural coupled simulation subjected to $30 \mathrm{~V}$ applied voltage and $22{ }^{\circ} \mathrm{C}$ sink temperature: (a) the lateral displacement of the micro griper tip in $\mathrm{mm}$, (b) the vertical displacement of the micro griper tip in $\mathrm{mm}$.

are Thermal-electric elements for the current path and Heat Transfer elements for the isolated tracks.

The second step is to use the temperature distribution that was computed in thermal-electric analysis in a thermomechanical analysis to provide a model of the deflection experienced by the gripper tip. For the thermo-mechanical analysis the 3D stress element was used. To keep the analysis simple the model assumed the gripper was made of one material only [19], rather than the actual, more complicated, structure consisting of polymer with a Platinum thin film coating. Therefore to reflect this, electrical material properties for the single material were modified to provide an average electrical resistance for the structure. For the case of thermal resistivity the model neglects the contribution of the platinum layer which is insignificant when compared to the bulk of the structure.

The exact thickness of the coating layer around the chevron was difficult to measure. Moreover, according to Maaroof [20], depositing very thin platinum films with different temperatures and deposition rates can lead to a change in the electrical resistivity of the deposited material. Therefore, to determine an effective resistivity for the structure, measurements were taken across the chevrons over the intended current path of an existing gripper. The approximated resistivity of the model can be calculated based on equation (1), where $R, w_{\mathrm{c}}, t_{\mathrm{c}}$ and $l_{\mathrm{c}}$ are the resistance of the chevron, width, thickness and the length of the chevron beam, respectively.

$$
\rho=R \frac{w_{\mathrm{c}} t_{\mathrm{c}}}{l_{\mathrm{c}}} .
$$

With the mean of measured resistance of $72 \mathrm{k} \Omega$, the crosssection area of $0.18 \times 1.44 \mathrm{~mm}^{2}$ and the length of beam of $3.46 \mathrm{~mm}$, the approximated resistivity used in the simulation was $\approx 5400 \Omega \mathrm{mm}$.

The power generated via joule heating at a certain temperature can be balanced with the heat conduction through the chevron structure and heat loss of the convection and radiation through the surface of the gripper device as described in equation (2) [21], Where $k_{\mathrm{pol}}, h_{\mathrm{conv}}, A_{\mathrm{s}}, h_{\mathrm{rad}}$ and $\Delta T$ are the thermal conductivity of the photopolymer, heat transfer 


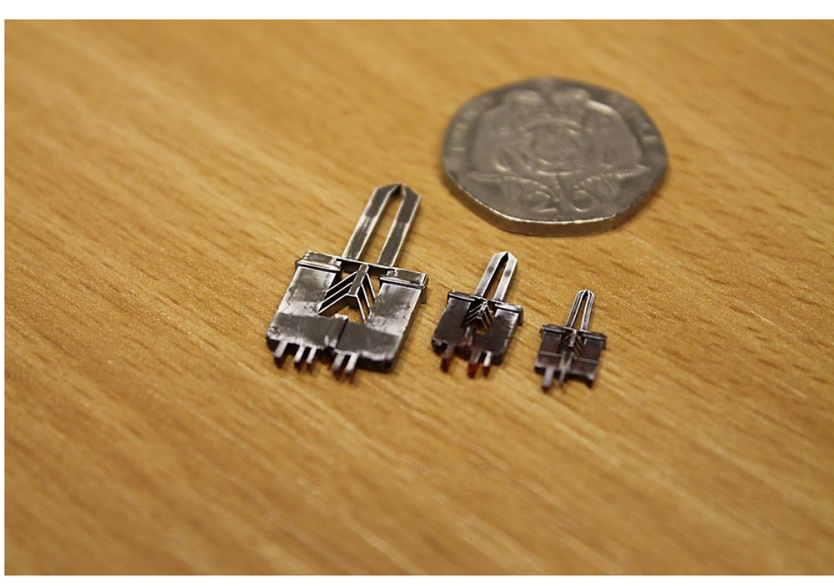

Figure 9. Metalized micro-gripper with chevron style micro thermal actuator.

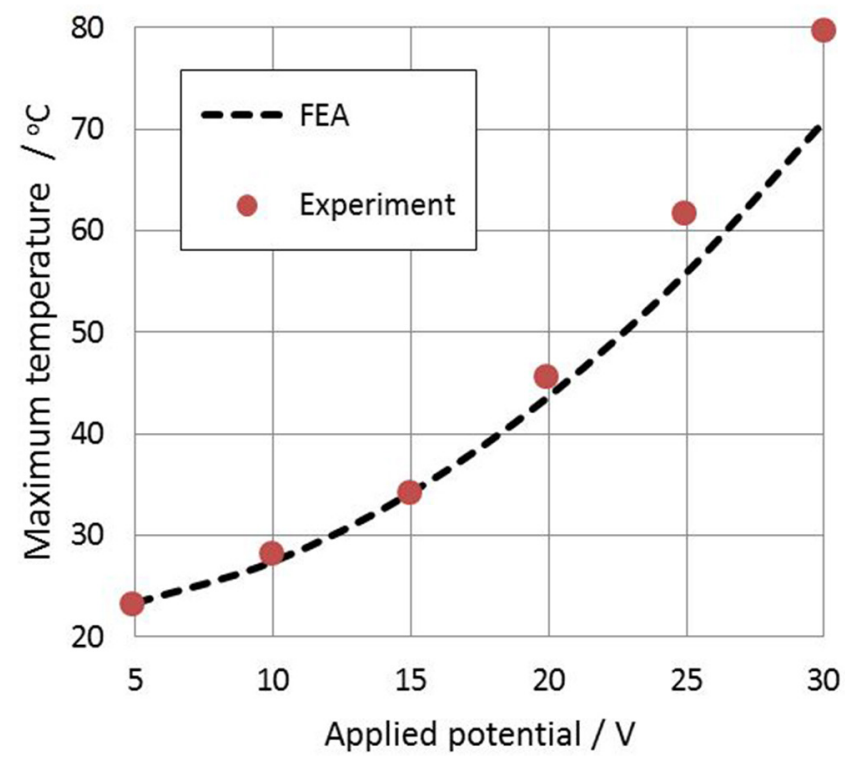

Figure 10. Experiment and simulation voltage-temperature curve.

coefficient via convection, surface area of chevrons, heat transfer coefficient via radiation and the temperature difference between the thermal actuator and ambient.

$$
P=\left[\frac{k_{\mathrm{pol}} w_{\mathrm{c}} t_{\mathrm{c}}}{l_{\mathrm{c}}}+h_{\mathrm{conv}} A_{\mathrm{s}}+h_{\mathrm{rad}} A_{\mathrm{s}}\right] \Delta T .
$$

In this work the effect of radiation was neglected, as it is small compared to convection and conduction at low operating temperatures (specified as less than $300{ }^{\circ} \mathrm{C}$ ) [22]. The surface film condition of $14\left(\mathrm{~W} \mathrm{~m}^{-2} \mathrm{~K}^{-1}\right)$ was implemented in the model to define the heat transfer coefficient $\left(h_{\text {conv }}\right)$ for free convection at the surface of thermal actuator. This value was calculated based on a ambient of $22{ }^{\circ} \mathrm{C}$ and a characteristic length of $7 \mathrm{~mm}$ [23].

To illustrate the modelling process, the electrical potential distribution over the micro-scale gripper is illustrated in figure 7(a). Applying the $30 \mathrm{~V}$ boundary condition to the first pad and making the second one equal to zero, causes current flow along the chevrons beams where the ambient temperature was assumed to be $22{ }^{\circ} \mathrm{C}$. This figure indicates that the

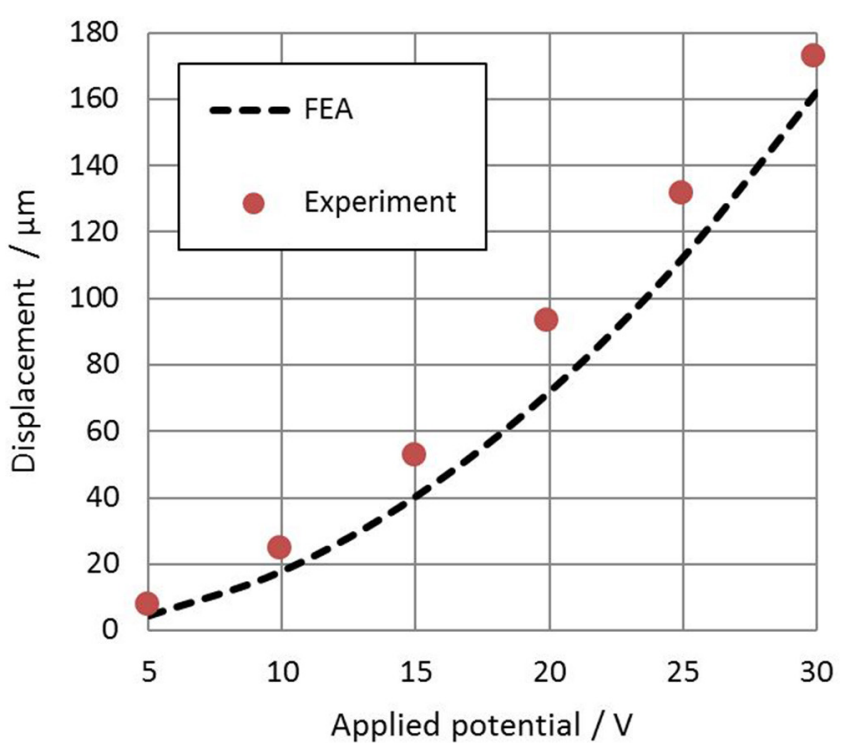

Figure 11. Graph plotting lateral displacement versus voltage for the micro-gripper tips.

voltage was reduced by half at the shuttle positioned at the centre of the chevron actuator. Thus, the applied potential drops between the two pads which lead to a rise in temperature and increased thermal load.

Figure 5(b) shows the temperature contour over the polymeric micro-gripper that results from the electrical current flow. It is clear that the temperature of the lower side of the shuttle was higher than the upper side by approximately $7{ }^{\circ} \mathrm{C}$ as result of the heat dissipation into the arms via conduction. Therefore, the two lower chevron beams expanded more than the top beams due to reaching the maximum temperature of $70{ }^{\circ} \mathrm{C}$.

Figure 8(a) shows the intended lateral displacement of the micro-gripper when driven at $30 \mathrm{~V}$, and it shows that the induced displacement of the gripper is a result of the expansion of the chevron beams; under this condition the maximum displacement between the gripper tips was approximately $160 \mu \mathrm{m}$. The deformed shape of the micro actuator in the vertical direction is shown in figure $8(\mathrm{~b})$.

\section{Experimental results and discussion}

The fabricated gripper can be seen in figure 9; this was activated by passing a current through the chevron actuator at the centre of the device. To test the micro-gripper it was located under a microscope to measure the lateral displacement at the tip of the gripper jaws as voltage was applied to the chevron heater circuit. Figure 10 shows the relationship between the voltage and maximum temperature for both experimental and simulation results, where the maximum temperature was obtained by using an infrared noncontact thermometer. It was found that the maximum temperature of the micro-gripper reached around $80{ }^{\circ} \mathrm{C}$ at $30 \mathrm{~V}$. This temperature did not adversely affect the gripper as the glass transmission temperature for the photopolymer (R11) is between 151 and $192^{\circ} \mathrm{C}$. Figure 11 shows the deflection of the micro-gripper for an 
applied voltage. A displacement of $8 \mu \mathrm{m}$ was measured for $5 \mathrm{~V}$ applied voltage, this indicates the polymeric micro-gripper can begin actuation at a low potential difference. The displacement continued to grow up to a maximum value of $182 \mu \mathrm{m}$.

Figures 10 and 11 also provide a comparison between the experimental results and those predicted using the finite element model. It can be easily observed that the experimental results differ from the finite element model results. Both predicted temperature and displacement were underestimated by the model. The reason for this error is most likely to be a result of incorrectly estimating the electrical properties of the metallic layer. In this work the resistivity of the micro-gripper was estimated by measuring the resistance between chevrons beams. However, the results are reasonable and the model is able to provide a basic approximation to the expected performance of the micro-gripper.

While the gripper was not designed to meet the needs of a specific application, the geometry and actuation performance of the gripper make it ideally suited to handling miniature submillimeter parts with sizes on the order of $600 \mu \mathrm{m}$. This is based on the current configuration of the actuators that start from a nominal open position of $540 \mu \mathrm{m}$, and extended to and opening of $700 \mu \mathrm{m}$. Using the flexibility provided by the additive manufacturing process this performance can easily be tailored to a give application. Inversion of the chevron actuators would reverse the direction of the grippers tending them to close instead of open; leading to a gripper with an initial opening of $540 \mu \mathrm{m}$ that reduces to $380 \mu \mathrm{m}$ when energized.

To further understand the limits of the process, the manufacturing processes used in this work must be considered. The 3D Projection-microstereolithography (PMSL) system used to manufacture the main structure of the gripper is limited to create layer based structures with a layer height of $25 \mu \mathrm{m}$. Within each layer the features can be defined with a pixel resolution of $10 \mu \mathrm{m}$. Therefore the capabilities of the manufacturing process place a limit on the ability to reduce in size of the micro gripper fingers to allow smaller objects to be handled. Using alternative and emerging processes will allow for smaller micro grippers or other devices to be fabricated. For example, advances in the application of two-photon polymerization techniques have made it possible to manufacture complex intricate micro parts with sub-micron feature sizes [6].

\section{Conclusions and outlook}

A novel and simple process to produce electrically active micro devices using a micro stereo lithography process has been developed. The capability of the process to fabricate complex devices, such as a micro thermal actuator has also been demonstrated. The process has significant levels of design freedom as it allows for true 3D geometries to be created with minimal design constraints. The process does not rely on any predefined photomasks or other device specific tooling, it therefore allows fast cost-effective production of prototype devices. Furthermore it results in an efficient design life cycle allowing test structures and prototypes to be quickly evaluated and modified such that an optimum structure may be converged upon. The process also allows cost effective production of highly specialized or customized devices as no investment in tooling of any kind is required which may limit design freedom. In addition it has been shown that the process is also compatible with computer simulation techniques based on finite element multi-physics simulation, which are commonly used in the design and development of traditional MEMS devices. The simulations undertaken as part of this work made use of relatively simple linear models, but were shown to be in good agreement with the experimental results achieved; both in terms of the overall behavior of the device as well as the absolute numerical results. While the process is limited in terms of being able to manufacture devices in high volumes it nevertheless represents an interesting option for the fabrication of highly customized devices, such as custom built micro-grippers for highly specialized micro-assembly jobs.

\section{Acknowledgments}

The authors wish to acknowledge the contribution of the Precision Manufacturing Centre at the University of Nottingham who supplied access to the equipment required to complete this work. In addition the authors would like to acknowledge the funding contribution from the Engineering and Physical Sciences Research Council of the UK for funding provided via the EPSRC Centre for Innovative Manufacturing in Intelligent Automation (grant EP/I033467/1).

\section{Author contributions}

Peter Kinnell created the concept and overall process, James Overton manufactured micro 3D printed devices and specimens, Khalid Alblalaihid designed and tested the microgripper and Simon Lawes contributed to the analysis of the experimental test results.

\section{References and notes}

[1] van Noort R et al 2012 The future of dental devices is digital Dent. Mater. 28 3-12

[2] Baufeld B, Brandl E and van der Biest O 2011 Wire based additive layer manufacturing: comparison of microstructure and mechanical properties of Ti-6Al-4V components fabricated by laser-beam deposition and shaped metal deposition J. Mater. Process. Technol. 211 1146-58

[3] Zhang X, Jiang X and Sun C 1999 Micro-stereolithography of polymeric and ceramic microstructures Sensors Actuators A 77 149-56

[4] Farsari M and Chichkov B N 2009 Industry perspective technology focus materials processing two-photon fabrication Nat. Photonics 3 450-2

[5] Lee K-S, Kim R H, Yang D-Y and Park S H 2008 Advances in 3D nano/microfabrication using two-photon initiated polymerization Prog. Polym. Sci. 33 631-81

[6] Vaezi M, Seitz H and Yang S 2013 A review on 3D microadditive manufacturing technologies Int. J. Adv. Manuf. Technol. 67 1721-54

[7] Che Harun F K, Taylor J E, Covington J A and Gardner J W 2009 An electronic nose employing dual-channel odour separation 
columns with large chemosensor arrays for advanced odour discrimination Sensors Actuators B 141 134-40

[8] Kang H-W, Lee I H and Cho D-W 2006 Development of a micro-bellows actuator using micro-stereolithography technology Microelectron. Eng. 83 1201-4

[9] Leigh S J, Purssell C P, Bowen J, Hutchins D A, Covington J A and Billson D R 2011 A miniature flow sensor fabricated by micro-stereolithography employing a magnetite/acrylic nanocomposite resin Sensors Actuators A 168 66-71

[10] Brkic B, France N, Clare A T, Sutcliffe C J, Chalker P R and Taylor S 2009 Development of quadrupole mass spectrometers using rapid prototyping technology $J$. Am. Soc. Mass Spectrom. 20 1359-65

[11] Ho K S, Bradley R J, Billson D R and Hutchins D A 2008 Micro-stereolithography as a transducer design method Ultrasonics 48 1-5

[12] Kinnell P K, Bail R, Alblalaihid K, Segal J and Ratchev S 2012 Rapid manufacturing of micro devices with integral electrical tracks Proc. Eng. 47 734-7

[13] Talib M 2012 Fabrication and characterisation of 3 dimensional scaffold for tissue engineering application via microstereolithography technique $P h D$ Thesis University of Warwick (http://webcat.warwick.ac.uk/ record $=\mathrm{b} 2581791 \sim \mathrm{S} 1$ )

[14] Madou M 2002 Fundamentals of Microfabrication: the Science of Miniaturization (Boca Raton, FL: CRC Press)

[15] Jain R K, Majumder S, Ghosh B and Saha S 2015 Design and manufacturing of mobile micro manipulation system with a compliant piezoelectric actuator based micro gripper J. Manuf. Syst. 35 76-91
[16] Kim K, Liu X, Zhang Y and Sun Y 2008 Nanonewton force-controlled manipulation of biological cells using a monolithic MEMS microgripper with two-axis force feedback J. Micromech. Microeng. 1855013

[17] Zhang Y, Chen B K, Liu X and Sun Y 2010 Autonomous robotic pick-and-place of microobjects IEEE Trans. Robot. 26 200-7

[18] Tai K, El-Sayed A-R, Shahriari M, Biglarbegian M and Mahmud S 2016 State of the art robotic grippers and applications Robotics 511

[19] Nguyen N-T et al 2004 A polymeric microgripper with integrated thermal actuators J. Micromech. Microeng. 14 969-74

[20] Maaroof A I and Evans B L 1994 Onset of electrical conduction in Pt and Ni films J. Appl. Phys. 76 1047-54

[21] Luo J K et al 2005 Comparison of microtweezers based on three lateral thermal actuator configurations J. Micromech Microeng. 15 1294-302

[22] Lau G K et al 2007 An in-plane thermal unimorph using confined polymers J. Micromech. Microeng. 17 S174-83

[23] Incropera F P and De Witt D P 1985 Fundamentals of Heat and Mass Transfer 2nd edn (Wiley)

[24] Iseki H 2001 Simplified FEM simulation of curl distortion in stereo-lithograpy with scanning laser beam Proc. of the 7th Int. Conf. Numerical Methods in Industrial Forming Processes pp 167-71

[25] Altun-Çiftçiouglu G A 2008 Mathematical modeling and simulation of photopolymerization process PhD Thesis Istanbul Technical University 